\title{
SIMILARITY TO CONTRACTION AND ASYMPTOTICS OF A CLASS OF INFINITE-DIMENSIONAL DISCRETE-TIME SYSTEMS
}

\author{
JOSEPH J. YAMÉ
}

Received 20 March 2004 and in revised form 25 June 2004

A class of infinite-dimensional discrete-time state operators is exhibited as concrete instances of power-bounded operators that are not similar to contractions. It is shown that such discrete-time systems arise from sampled feedback control of unstable continuoustime systems. The asymptotic behavior of the state operators of these discrete systems is not intimately related to their spectral radius.

\section{Introduction}

This note is originally motivated by the stability property of continuous-time dynamical systems controlled by digital processors within the lifting framework as exposed in [2]. Our purpose is to exhibit a result on stability which seems counterintuitive, at least for most control engineers, and at the same time serves as a practical and simple engineering counterexample to an important question raised by Sz.-Nagy in the late fifties. This question is whether every power-bounded (p.w.b.) operator is similar to a contraction (s.c.). This mathematical problem which was initially formulated as a conjecture was proven wrong by Foguel [3] and Halmos [5]. Halmos reformulated it in 1970 in a more sophisticated way as [7]: is every polynomially bounded (p.b.) operator s.c.? A negative answer to Halmos question appeared only recently and is due to Pisier [11] who gave an ingenious example of an operator that is p.b. but not s.c. The abstract nature of the construction of the counterexamples in the mathematical literature might lead to think that these operators are esoteric and might rarely, or even cannot, occur in real engineering problems. When this occurs, it is quite exciting to realize that an abstract result in a specific class of pure mathematical problems is shared by fields of engineering and this, in turn, gives some hope that exchanging ideas between the fields might lead to new insights on the original problems as well as deepening our understanding of its engineering counterpart. The relevance of Sz.-Nagy question to control engineering analysis problems lies in the asymptotic behaviors of discrete-time systems, such asymptotics being usually determined in terms of contractiveness (or noncontractiveness) of the state-transition operators of these dynamical systems. The standard mathematical tool for control engineers analyzing the behavior of an evolving dynamical system is the spectrum (or more 
specifically the eigenvalues) of the operator (or matrix) governing the dynamics of the system. Such spectra supplement the abstract notion of an operator by a visual information in the complex plane, and their analysis has proved highly successful in applications. For discrete-time systems, it is well known that when the spectrum of the state operator is contained in the open unit disc of the complex plane, which means also that the state operator is a strict contraction, the norms of the iterates of the state operator converge to the zero operator. Such convergence in the operator norm is called uniform stability or exponential stability and it implies also that the state trajectory converges to zero. When the state operator has at least a spectral value outside the closed unit disc, which means that the state operator is not a contraction, the norms of the iterates of the state operator diverge to infinity and in that case the state trajectory is known to diverge at least for finite-dimensional systems. The convergence of the state to zero is called asymptotic stability and it is well known that the notions of exponential and asymptotic stabilities are actually equivalent for linear systems in finite-dimensional spaces. This has given to control engineers the "feeling" that when a discrete dynamical system is stable (in the sense of asymptotic stability), its spectral values are at most contained in the closed unit disc and if this is not the case, then the system is unstable. This note shows that feedback control can be used to generate very common system operators that are asymptotically stable albeit their spectrum is not a subset of the closed unit disc. The link to Sz.-Nagy's question is obtained via a trivial connection between asymptotic stability and power-boundedness of the state operator and thus yielding concrete and simple counterexamples of p.w.b. operators which are not similar to contractions.

\section{Preliminary}

Let $\mathfrak{X}$ and $Y$ denote separable Hilbert spaces and $\mathscr{B}(\mathfrak{X}, Y)$ the set of all bounded linear operators from $\mathfrak{X}$ to $Y$. The set $\mathscr{B}(\mathfrak{X}, Y)$ will be denoted simply by $\mathscr{B}(\mathfrak{X})$ when $\mathfrak{X}=Y$. A norm on a space $Z$ will be denoted by $\|\cdot\|_{Z}$. An operator $T \in \mathscr{B}(\mathfrak{X})$ is said to be p.b. if there exists a constant $M$ such that

$$
\|p(T)\|_{\mathscr{B}(\mathfrak{X})}<M\|p\|_{\infty}
$$

for any scalar polynomial $p$, where

$$
\|p\|_{\infty}=\max \{|p(z)|:|z|=1\}
$$

is the so-called infinity norm of the analytic polynomial $p$. When the polynomial in (2.1) is of the form $p(z)=z^{n}$, where $n$ is a positive integer, then $T \in \mathscr{B}(\mathfrak{X})$ is said to be p.w.b., that is,

$$
\sup _{n \in \mathbb{Z}_{+}}\left\|T^{n}\right\|_{\mathscr{B}(\mathfrak{X})}<+\infty,
$$

or equivalently, the sequence $\left\{T^{n}\right\}_{n \geq 0}$ is a bounded sequence of operators. Recall that a contraction is an operator $C \in \mathscr{B}(\mathfrak{X})$ such that $\|C\|_{\mathscr{B}(\mathfrak{X})} \leq 1$ and a strict contraction is an operator $C \in \mathscr{B}(\mathfrak{X})$ such that $\|C\|_{\mathscr{B}(\mathfrak{X})}<1$. An operator $T$ is s.c. if there is a boundedly 
invertible operator $S \in \mathscr{B}(\mathfrak{X})$, with $\left\|S^{-1}\right\|_{\mathscr{B}(\mathfrak{X})}<\infty$, such that

$$
\left\|S^{-1} T S\right\|_{\mathscr{B}(\mathfrak{X})} \leq 1 .
$$

The operators $T$ and $S^{-1} T S$ are then said to be similar. Let $\sigma(T)$ denote the spectrum of operator $T$, then the spectral radius of operator $T$ is the positive real number defined by

$$
r_{\sigma}(T)=\lim _{n \rightarrow \infty}\left\|T^{n}\right\|_{\mathscr{B}(\mathfrak{X})}^{1 / n}=\max _{\lambda \in \sigma(T)}|\lambda| .
$$

Similarity is an equivalence relation which preserves each part of the spectrum and hence the spectral radius [6, Corollary 4, page 77], that is, $r_{\sigma}(T)=r_{\sigma}\left(S^{-1} T S\right)$. By virtue of Von Neumann's inequality, every contraction in $\mathscr{B}(\mathfrak{X})$ satisfies inequality (2.1) with $M=1$ as well as inequality (2.3) and the following implications hold:

$$
T \text { is s.c. } \Longrightarrow T \text { is p.b. } \Longrightarrow T \text { is p.w.b. }
$$

Thus, one may consider the class of p.b. (and hence the class of p.w.b.) operators as a generalization of the class of contraction operators in $\mathscr{B}(\mathfrak{X})$ and this was the motivation of Sz.-Nagy for asking if the reverse implication holds. It is worth noticing that for operators on finite-dimensional spaces all these notions are equivalent and Sz.-Nagy proved more than that: every p.w.b. compact operator is s.c. This raised the question: does the equivalence between power-boundedness and similarity to a contraction still hold for noncompact operators?

\section{Abstract discrete representation of continuous-time systems}

Consider the following $n$-dimensional homogeneous continuous-time time-invariant system:

$$
\dot{x}(t)=A x(t) .
$$

On the time interval $[0, \infty)$, the continuous-time state trajectory of this system satisfies

$$
x(t+h)=\Phi x(t), \quad \forall t \in[0, \infty),
$$

where $h$ is a fixed positive real number and $\Phi=e^{A h}$. An abstract version of the above equation is obtained by introducing the mapping $\mathscr{F}: \mathfrak{X} \rightarrow \mathfrak{X}$,

$$
x_{k} \longmapsto x_{k+1}=\mathscr{F} x_{k},
$$

which is defined pointwise by

$$
\left(\mathscr{F} x_{k}\right)(t)=\Phi x_{k}(t), \quad \forall t \in[0, h),
$$

and where we have set

$$
x_{k}(t)=x(t+k h) \quad \text { for } t \in[0, h), k \in \mathbb{N}
$$


and $\mathfrak{X}=L^{2}\left([0, h), \mathbb{R}^{n}\right)$. Clearly, the operator $\mathscr{F}$ is bounded on $\mathfrak{X}$. The $x_{k}$ 's are pieces of continuous functions on time intervals of length $[0, h)$. Note that the continuous-time dynamics of the original system is entirely embedded into the discrete-time dynamics (3.3). An important property of $\mathscr{F}$ is that its spectral values are the eigenvalues of the matrix $\Phi$, but each spectral value has an infinite geometric multiplicity. This property is easily seen by realizing $\mathfrak{X}$ as an $\aleph_{0}$-fold copies of $\mathbb{R}^{n}$, that is, $\mathfrak{X}$ is isomorphic to $\ell_{+}^{2}\left(\mathbb{R}^{n}\right)$, in which case $\mathscr{F}$ can be identified in a natural way with an infinite-dimensional matrix with the matrices $\Phi$ as its diagonal entries. This is stated precisely in the next theorem.

THeOREM 3.1. Each point in the spectrum $\sigma(\mathscr{F})$ is an eigenvalue of infinite multiplicity.

Proof. Let $\lambda_{i}$ be an eigenvalue of $\mathscr{F}$, then there exists a nonzero vector $x_{i} \in \mathfrak{X}$ such that

$$
\mathscr{F} x_{i}=\lambda_{i} x_{i}
$$

From the definition of operator $\mathscr{F}$, it is immediately seen that there exists a nonzero vector $u_{i} \in \mathbb{C}^{n}$ such that

$$
\Phi u_{i}=\lambda_{i} u_{i}
$$

Now, for any scalar function $\psi \in L_{2}[0, T)$, we have

$$
\Phi \psi(t) u_{i}=\lambda_{i} \psi(t) u_{i}, \quad \forall t \in[0, T),
$$

that is, the function $\phi_{i}=\psi u_{i} \in \mathfrak{X}$, defined by $\phi_{i}(t)=\psi(t) u_{i}$ for $0 \leq t<T$, is an eigenvector of operator $\mathscr{F}$ corresponding to the eigenvalue $\lambda_{i}$. Since $L_{2}[0, T)$ is a Hilbert space, it has an orthonormal basis, say $\left\{\psi_{n}\right\}_{n=1}^{\infty}$, and clearly the following holds true:

$$
\overline{\operatorname{span}}\left\{\psi_{n} u_{i}\right\}_{n=1}^{\infty} \subseteq \mathbf{E}_{\lambda_{i}},
$$

hence

$$
\operatorname{dim} \overline{\operatorname{span}}\left\{\psi_{n} u_{i}\right\}_{n=1}^{\infty}=\aleph_{0} \leq \operatorname{dim} \mathbf{E}_{\lambda_{i}},
$$

where $\mathbf{E}_{\lambda_{i}}=\operatorname{ker}\left(\lambda_{i} I-\mathscr{F}\right)$. The eigensubspace $\mathbf{E}_{\lambda_{i}}$ being a subspace of the separable space $\mathfrak{X}, \operatorname{dim} \mathbf{E}_{\lambda_{i}}$ cannot exceed $\kappa_{0}$, and therefore

$$
\operatorname{dim} \mathbf{E}_{\lambda_{i}}=\aleph_{0} .
$$

Now, return to the original system and fix $n=1$ for simplicity of the analysis in the sequel. Assume that the 1-dimensional differential equation (3.1) is perturbed by a scalar function, that is, $\dot{x}=A x(t)+u(t)$, where the perturbation $u$ is a control input obtained by sampled-state feedback with a zero-order hold, that is,

$$
u(t)=K x(k h) \quad \text { for } t \in[k h, k h+h)
$$

and $K$ is a feedback gain. Then, the closed-loop system is fully described by

$$
\dot{x}(t)=A x(t)+K x(k h) \quad \text { for } t \in[k h, k h+h) .
$$


From the abstract model (3.3) of the homogeneous system and the principle of superposition, the continuous-time state trajectory of system (3.13) can be lifted to a sequence $\left\{x_{k}\right\}_{k \geq 0}$, where each function piece $x_{k} \in \mathfrak{X}$ is given by

$$
x_{k+1}=\mathscr{F} x_{k}+p_{k} .
$$

The vector $p_{k} \in \mathfrak{X}$ is the perturbation resulting from the sampled-state feedback which can be described by a mapping $\mathscr{B}_{K}: \mathbb{R} \rightarrow \mathfrak{X}$, that is,

$$
p_{k}(t)=\left(\mathscr{B}_{K} x(k h)\right)(t) \quad \text { for } t \in[k h, k h+h) .
$$

It is easily shown that operator $\mathscr{T}_{K}$ is a bounded integral operator, however its expression is irrelevant here. Since $x(k h)$ is the sampled value of the continuous-time state at time $t=k h$, it can be seen as resulting from an operator $\widetilde{\mathscr{C}}_{1}$ on $\mathfrak{X}$. However, this operator $\widetilde{\mathscr{C}}_{1}$ is unbounded on $\mathfrak{X}$ since it is a sampling process which corresponds to point sensing in $\mathfrak{X}$. Indeed, the observation process (i.e., the sampling process) is described by

$$
\mathfrak{X} \supset \mathfrak{D}\left(\tilde{\mathscr{C}}_{1}\right) \stackrel{\tilde{\mathscr{C}}_{1}}{\longrightarrow} Y=\mathbb{R}
$$

with $\tilde{\mathscr{C}}_{1}$ defined by

$$
\phi[k]=\tilde{\mathscr{C}}_{1} q_{k} \triangleq \int_{0}^{T} \delta(\tau) q_{k}(\tau) d \tau=q_{k}(0)
$$

for $q_{k} \in \mathfrak{D}\left(\tilde{\mathscr{C}}_{1}\right)$ and where $\delta$ is the Dirac distribution. The observation operator $\tilde{\mathscr{C}}_{1}$ is actually an evaluation operator at the point $t=0$ of the distributed domain $[0, T)$. Clearly, $\tilde{\mathscr{C}}_{1}$ is not defined on all of $\mathfrak{X}$, its largest domain $\mathfrak{D}\left(\tilde{\mathscr{C}}_{1}\right)$ in which it is well defined is the subset of $\mathfrak{X}$ consisting of functions which are continuous on $[0, T)$. Moreover, this operator is unbounded on its domain since if we choose a sequence $\left\{q_{k}\right\}_{k \in \mathbb{N}}$ in $\mathfrak{D}\left(\tilde{\mathscr{C}}_{1}\right)$ with decreasing supports $\left[0, T_{k}\right) \subset[0, T)$, we can make $\left\|\tilde{\mathscr{C}}_{1} q_{k}\right\|_{Y}$ as large as we like, while keeping the norm $\left\|q_{k}\right\|_{\mathfrak{X}}=1$. In order to overcome these difficulties, we will show that $\tilde{\mathscr{C}}_{1}$ is an admissible observation operator [12] for the discrete-time infinite-dimensional system so that one can define a well-posed observation process.

\section{Admissibility of the observation operator}

We introduce the following definition of admissibility for discrete-time systems which is adapted from $[12,14]$ for continuous-time semigroup systems.

Definition 4.1. Let $Z$ denote a Hilbert space and suppose that $\mathbb{F}=\left\{\mathscr{F}^{n}\right\}_{n \geq 0}$ is a discrete semigroup on $Z$. Let $\mathscr{W}$ be a dense $\mathbb{F}$-invariant subspace of $Z$ and let the space of observations $Y$ be another Hilbert space. Then, $\mathscr{b}: \mathscr{W} \rightarrow Y$ is an admissible observation operator for $\mathbb{F}$ with respect to $\mathcal{W}$ if for any $m>0$, the observation map $O_{m}: \mathcal{W} \longrightarrow \ell_{2}(Y)$ defined by $O_{0}=0$ and

$$
\mathfrak{O}_{m} q=\left\{\mathscr{C} q, \mathscr{C} \mathscr{F} q, \mathscr{C}_{F}^{2} q, \ldots, \mathscr{C} \mathscr{F}^{m-1} q, 0,0,0, \ldots\right\}
$$

has a continuous extension to $Z$. 
In other words, admissibility of $\mathscr{C}$ means that for some integer $m>0$, and some real number $\kappa(m) \geq 0$ and any $q \in \mathcal{W}$,

$$
\sum_{n=0}^{m-1}\left\|\mathscr{G} \mathscr{F}^{n} q\right\|_{Y}^{2} \leq \kappa(m) \cdot\|q\|_{Z}^{2} .
$$

Inequality (4.2) expresses a continuity property of the observations with respect to initial conditions, that is, the observation map is bounded on $Z$.

For the well-posedness of the observation process, the problem is to find the right choice of $\mathcal{W}$ so that the observation operator satisfies the admissibility conditions. We introduce the Sobolev space $W^{1,2}[1]$ :

$$
\mathscr{W}=W^{1,2}([0, T), \mathbb{R})=\left\{x \in \mathfrak{X}: x \in \mathscr{A}_{c}([0, T), \mathbb{R}), \dot{x} \in \mathfrak{X}\right\},
$$

where $A_{c}([0, T), \mathbb{R})$ is the set of absolutely continuous functions on $[0, T)$ with values in $\mathbb{R}$, that is, the set of functions $x:[0, T) \rightarrow \mathbb{R}$ which have almost everywhere a derivative $\dot{x} \in L_{2}([0, T), \mathbb{R})$ and such that

$$
x(t)=\int_{0}^{t} \dot{x}(\xi) d \xi+x(0) \quad(\text { a.e. }) .
$$

The space $\mathcal{W}$ is in its own right a Hilbert space under the inner product

$$
\left\langle x_{1}, x_{2}\right\rangle_{\mathscr{W}}=\left\langle x_{1}, x_{2}\right\rangle_{\mathfrak{X}}+\left\langle\dot{x}_{1}, \dot{x}_{2}\right\rangle_{\mathfrak{X}},
$$

but note that $\mathscr{W}$ is not closed under the norm of $\mathfrak{X}$. Clearly, $\mathscr{W} \subset \mathfrak{X}$ with continuous injection, that is, the identity map $i: \mathscr{W} \rightarrow \mathfrak{X}$ is bounded, and it is in fact a dense subspace of $\mathfrak{X}$ with respect to the norm of $\mathfrak{X}$. If $q_{n} \in \mathcal{W} \subset \mathfrak{D}\left(\tilde{\mathscr{C}}_{1}\right)$ for some $n \geq 0$, then the operator $\tilde{\mathscr{C}}$ defined by

$$
\phi[n]=\tilde{\mathscr{C}} q_{n}=q_{n}(0)
$$

makes sense. From the definition of the semigroup generator $\mathscr{F}$ and the properties of elements in the subspace $\mathcal{W}$, it is easily verified that $\mathscr{F} \mathcal{W} \subset \mathcal{W}$ so that $\mathcal{W}$ is $\mathbb{E}$-invariant. Thus, for $q_{0} \in \mathcal{W}$, the formula

$$
\phi[n]=\tilde{\mathscr{C}}_{\mathscr{F}}{ }^{n} q_{0}
$$

has a well-defined meaning and allows us to define for any integer $m \geq 0$ the map $O_{m}$ : $W \rightarrow \ell_{2}(Y)$,

$$
\sigma_{m} q_{0}=\left\{\tilde{\mathscr{C}} q_{0}, \tilde{\mathscr{C}} \mathscr{F} q_{0}, \tilde{\mathscr{C}} \mathscr{F}^{2} q_{0}, \ldots, \tilde{\mathscr{C}} \mathscr{F}^{m-1} q_{0}, 0,0,0, \ldots\right\}
$$

Clearly, $\mathrm{O}_{m}$ is continuous on $\left(\mathcal{W},\|\cdot\|_{\mathfrak{X}}\right)$, and therefore the operators $\mathrm{O}_{m}$ from $\mathcal{W}$ to $\ell_{2}(Y)$ have unique continuous extensions to continuous operators from the closure of $\mathcal{W}$ in $\mathfrak{X}$, that is, $\overline{\left(\mathscr{W},\|\cdot\|_{\mathfrak{X}}\right)}=\mathfrak{X}$, to $\ell_{2}(Y)$. We denote this extension by the same symbol and we can write, therefore,

$$
\phi[n]=\left(O_{m} q_{0}\right)[n] \quad \text { for } n<m
$$


when $q_{0}$ is a function in $\mathfrak{X}$. We conclude that any finite-dimensional continuous-time system whose state is sampled can be represented by an infinite discrete-time system on $\mathfrak{X}$ and the sampling operator is an admissible observation operator for $\left\{\mathscr{F}^{n}\right\}_{n \in \mathbb{N}}$.

Next, it is easy to see that the following functional equation holds on $\ell_{2}(Y)$ :

$$
\mathrm{O}_{m+n} q=\mathrm{O}_{m} q^{m} \diamond_{m}\left(\mathscr{F}^{m} q\right)
$$

where the symbol $\stackrel{m}{\diamond}$ denote the concatenation operator defined by

$$
(\phi \diamond \stackrel{m}{\diamond})[n]= \begin{cases}\phi[n] & \text { for } n=0,1, \ldots, m-1, \\ \psi[n-m] & \text { for } n=m, m+1, \ldots\end{cases}
$$

If $n=0$ in the functional equation (4.10), then for any $m \geq 0$ we get that

$$
\mathrm{O}_{m}=P_{m} \mathrm{O}_{m}
$$

and for $m \leq k$,

$$
\mathrm{O}_{m}=P_{m} \mathrm{O}_{k}
$$

where $P_{m}$ is the projection operator defined by

$$
\phi \longmapsto \psi=P_{m} \phi \text { with } \begin{cases}\psi[n]=\phi[n] & \text { for } n=0,1, \ldots, m-1, \\ \psi[n]=0 & \text { for } n \geq m .\end{cases}
$$

At this stage, to simplify the analysis to follow, we assume that the semigroup $\mathbb{F}$ is strongly stable, meaning that $\lim _{n \rightarrow \infty}\left\|\mathscr{F}^{n} q\right\|_{\mathscr{X}} \rightarrow 0$ for all $q \in \mathscr{X}$. This hypothesis is not restrictive for the result to be derived, the general case can be treated along the same lines at the expense of some technicalities (see [14]). Under the above assumption, it is clearly seen that the operator $O_{\infty} \in \mathscr{B}\left(\mathfrak{X}, \ell_{2}(Y)\right)$ defined by

$$
\mathrm{O}_{\infty} q=\lim _{m \rightarrow \infty} \mathrm{O}_{m} q
$$

exits, and for any $m \geq 0$, equation (4.13) extends to

$$
\mathrm{O}_{m}=P_{m} \mathrm{O}_{\infty}
$$

Combining (4.10) and (4.16) yields

$$
\mathrm{O}_{\infty} q=\mathrm{O}_{\infty} q \diamond{ }^{m} \mathrm{O}_{\infty}\left(\mathscr{F}^{m} q\right)
$$

We define the operator $\Xi_{z}: \mathfrak{X} \rightarrow Y$ by the series

$$
\Xi_{z} q=\sum_{k=0}^{\infty} z^{-k}\left(\bigcirc_{\infty} q\right)[k]
$$


For $z \in \mathbb{C}$ such that $|z|>\|\mathscr{F}\|_{\mathscr{B}(\mathfrak{X})}$ or $|z|$ sufficiently large and from Lemma 7.1 (see the appendix), it is easily verified that this series is absolutely convergent and $\Xi_{z} \in \mathscr{B}(\mathfrak{X}, Y)$. For any $q \in \mathfrak{X}$, the series can be decomposed as

$$
\begin{aligned}
& \Xi_{z} q=\sum_{k=0}^{m-1} z^{-k}\left(\bigcirc_{\infty} q\right)[k]+\sum_{k=m}^{\infty} z^{-k}\left(\mathcal{O}_{\infty} \mathscr{F}^{m} q\right)[k-m], \\
& \Xi_{z} q=\sum_{k=0}^{m-1} z^{-k}\left(\bigcirc_{\infty} q\right)[k]+z^{-m} \Xi_{z} \mathscr{F}^{m} q
\end{aligned}
$$

and we have

$$
\sum_{k=0}^{m-1} z^{-k}\left(O_{\infty} q\right)[k]=\Xi_{z} q-z^{-m} \Xi_{z} \mathscr{F}^{m} q .
$$

For $m=1$ and any $q \in \mathfrak{X},(4.20)$ leads to

$$
\left(0_{\infty} q\right)[0]=\Xi_{z} q-z^{-1} \Xi_{z} \mathscr{F} q
$$

In view of the expression (4.8) of the observation map, we set

$$
\tilde{\mathscr{C}}_{1}^{a} q=\left(0_{\infty} q\right)[0]
$$

and since $\mathscr{F} \in \mathscr{B}(\mathfrak{X})$, we get from $(4.21)$ that $\tilde{\mathscr{C}}_{1}^{a} \in \mathscr{B}(\mathfrak{X}, Y)$ and is given by

$$
\begin{aligned}
& \tilde{\mathscr{C}}_{1}^{a} q=z^{-1} \Xi_{z}\left(z I-\mathscr{F}^{\prime}\right) q, \\
& \Xi_{z} w=z \tilde{\mathscr{C}}_{1}^{a}(z I-\mathscr{F})^{-1} w .
\end{aligned}
$$

The operator $\tilde{\mathscr{C}}_{1}^{a}$ defined by (4.23), which we call the abstract observation operator, is a bounded operator defined on all of $\mathfrak{X}$ and its significance is that it is now possible to give an interpretation of the observation equation for any $q_{n}$ in the state-space $\mathfrak{X}$, whereas the original operator $\tilde{\mathscr{C}}_{1}$ in $(3.17)$ is unbounded on $\mathfrak{X}$. The above result holds also true if the semigroup $\mathbb{F}$ was not assumed to be strongly stable. It is interesting to compare this result with the "regularization" of the sampling process with a prefilter placed before the sampling operator as described by [2] in an input/output setting.

Since $Y=\mathbb{R}$, the abstract observation operator $\tilde{\mathscr{C}}_{1}^{a}$ is actually a linear functional on the Hilbert space $\mathfrak{X}$. Then, from the Riesz representation theorem [6], there exists a unique element $c_{1} \in \mathfrak{X}$ such that

$$
\phi[n]=\tilde{\mathscr{C}}_{1}^{a} q_{n}=\left\langle c_{1}, q_{n}\right\rangle_{\mathfrak{X}}
$$

and the norm of the operator $\tilde{\mathscr{C}}_{1}^{a}$ is simply given by

$$
\left\|\tilde{\mathscr{C}}_{1}^{a}\right\|_{\mathscr{B}(\mathfrak{X}, Y)}=\left\|c_{1}\right\|_{\mathfrak{X}} .
$$


It is interesting to note that, as a bounded operator, the observation map $\tilde{\mathscr{C}}_{1}^{a}$ admits an adjoint operator $\widetilde{\mathscr{C}}_{1}^{a *}$ which is easily computed as

$$
\begin{aligned}
\left\langle\tilde{\mathscr{C}}_{1}^{a} q_{n}, \psi[n]\right\rangle_{\mathbb{R}} & =\left\langle q_{n}, \tilde{\mathscr{C}}_{1}^{a *} \psi[n]\right\rangle_{\mathfrak{X}} \\
& =\int_{0}^{T} q_{n}^{*}(\tau)\left(\tilde{\mathscr{C}}_{1}^{a *} \psi[n]\right)(\tau) d \tau \\
& =\overline{\left\langle c_{1}, q_{n}\right\rangle_{\mathfrak{X}}} \cdot \psi[n] \\
& =\int_{0}^{T}\left\{c_{1}^{*}(\tau) q_{n}(\tau)\right\}^{*} d \tau \psi[n] \\
& =\int_{0}^{T} q_{n}^{*}(\tau) c_{1}(\tau) \psi[n] d \tau,
\end{aligned}
$$

that is, the operator $\tilde{\mathscr{C}}_{1}^{a *}: \mathbb{R} \rightarrow \mathfrak{X}$ is given by

$$
\left(\tilde{\mathscr{C}}_{1}^{a *} \psi[n]\right)(\tau)=c_{1}(\tau) \psi[n], \quad 0 \leq \tau<T
$$

The adjoint $\tilde{\mathscr{C}}_{1}^{a *}$ of the abstract observation map behaves like a generalized hold function [9].

\section{A counterexample to Sz.-Nagy problem using feedback control}

The observation process on $\mathfrak{X}$ resulting from sampling can now be written as

$$
\tilde{\mathscr{C}}_{1}^{a}: \mathfrak{X} \longrightarrow \mathbb{R} \quad \text { with } \tilde{\mathscr{C}}_{1}^{a} x_{k}=x(k h)
$$

with $\tilde{\mathscr{C}}_{1}^{a}$ bounded on $\mathfrak{X}$. Combining (3.15) and (5.1), the closed-loop state trajectory $\left\{x_{k}\right\}_{k \geq 0}$ of the original system (3.13) is given by the abstract discrete-time autonomous equation on $\mathfrak{X}$,

$$
x_{k+1}=\left(\mathscr{F}+\mathscr{B}_{K} \tilde{\mathscr{C}}_{1}^{a}\right) x_{k}
$$

From now, we assume that the sampled-state feedback is stabilizing in the sense that the closed-state trajectory converges asymptotically to zero, that is,

$$
\lim _{n \rightarrow \infty}\left\|\left(\mathscr{F}+\mathscr{\mathscr { B }}_{K} \tilde{\mathscr{C}}_{1}^{a}\right)^{k} x_{0}\right\|_{\mathscr{X}}=0, \quad \forall x_{0} \in \mathfrak{X}
$$

Note that this is always possible under some mild conditions [4] which reduce to a controllability condition of the sampled model of (3.1) and a nonpathological condition on the sampling frequency. Under these mild conditions, the asymptotic stability of the feedback system (3.13) is guaranteed by choosing the feedback gain $K$ such that the state matrix (here, a scalar) $(\Phi+K)$ of its stroboscopic model (i.e., its sampled model) is a stable matrix. Then, we have the following result. 
Proposition 5.1. Under the stabilizing assumption of the sampled-state feedback, the closed-loop state operator is p.w.b., that is,

$$
\sup _{k \geq 1}\left\|\left(\mathscr{F}+\mathscr{B}_{K} \tilde{\mathscr{C}}_{1}^{a}\right)^{k}\right\|_{\mathscr{B}(\mathscr{X})}<\infty
$$

Proof. Since the operator $\mathscr{F}+\mathscr{G}_{K} \tilde{\mathscr{C}}_{1}^{a}$ is bounded on $\mathfrak{X}$, the result is a direct application of the uniform boundedness principle [6].

Now, consider a continuous-time system (3.1) whose abstract discrete open-loop state operator, denoted by $\mathscr{F}_{u}$, is such that

$$
\sigma\left(\mathscr{F}_{u}\right) \cap \mathbb{D}^{c} \neq \varnothing,
$$

where

$$
\mathbb{D}^{c}=\{z \in \mathbb{C}:|z|>1\}
$$

Such system is an unstable open-loop system. The following slightly disappointing result holds.

Proposition 5.2. The closed-loop state feedback operator $\left(\mathscr{F}_{u}+\mathscr{B}_{K} \tilde{\mathscr{C}}_{1}^{a}\right)$ is p.w.b. but not s.c.

Proof. The power-boundedness results from Proposition 5.1. Since $\sigma\left(\mathscr{F}_{u}\right) \cap \mathbb{D}^{c} \neq \varnothing$, there is at least one element $\lambda \in \sigma\left(\mathscr{F}_{u}\right)$ such that $|\lambda|>1$. For operator $\tilde{\mathscr{C}}_{1}^{a}$ having a finite range, the perturbation $\mathscr{B}_{K} \tilde{\mathscr{C}}_{1}^{a}$ is compact. From the compactness of operator $\mathscr{B}_{K} \widetilde{\mathscr{C}}_{1}^{a}$ and the fact that all spectral values of $\mathscr{F}_{u}$ have infinite multiplicity, the following inclusion holds [10]:

$$
\sigma\left(\mathscr{F}_{u}\right) \subset \sigma\left(\mathscr{F}_{u}+\mathscr{B}_{K} \tilde{\mathscr{C}}_{1}^{a}\right)
$$

which implies that the spectrum of $\mathscr{F}_{u}+\mathscr{\mathscr { B }}_{K} \tilde{\mathscr{C}}_{1}^{a}$ has an element $\lambda \in \mathbb{C}$ such that $|\lambda|>1$ and therefore its spectral radius

$$
r_{\sigma}\left(\mathscr{F}_{u}+\mathscr{B}_{K} \tilde{\mathscr{C}}_{1}^{a}\right)=\sup _{\lambda \in \sigma\left(\mathscr{F}_{u}+\mathscr{B}_{K} \tilde{\mathscr{C}}_{1}^{a}\right)}|\lambda| \geq 1
$$

Since similarity preserves the spectral radius [6, Corollary 4, page 77], the operator $\mathscr{F}_{u}+$ $\mathscr{B}_{K} \tilde{\mathscr{C}}_{1}^{a}$ on $\mathfrak{X}$ is clearly not s.c.

Actually, such an operator may be arbitrarily far from being similar to contractions. It is interesting to note that from the spectral mapping theorem the operator $\mathscr{F}_{u}+\mathscr{B}_{K} \tilde{\mathscr{C}}_{1}^{a}$ 
cannot converge uniformly to zero, that is,

$$
\lim _{n \rightarrow \infty}\left\|\left(\mathscr{F}+\mathscr{B}_{K} \tilde{\mathscr{C}}_{1}^{a}\right)^{k}\right\|_{\mathscr{B}(\mathscr{C})} \neq 0 .
$$

We conclude that unstable continuous-time systems under stabilizing sampled-state feedback control have closed-loop lifted state operators which are p.w.b. without being similar to contractions. Many feedback control systems fall in this category, and contrary to the pure abstract mathematical constructions $[3,5]$, the feedback operators of such systems are concrete and simple physical counterexamples to Sz.-Nagy's question. Such systems are in fact asymptotically stabilizable, but never uniformly stabilizable on $\mathfrak{X}$. The counterexample presented in this paper is instructive for identifying the main mathematical properties of a more general class of discrete-time systems which might lead to noncontractive operators which are p.w.b. This is an ongoing research topic.

\section{A remark}

The negative answer to the similarity problem raised in this paper shows that the spectra of operators (preserved under similarity transformations) are not always good indicators of their asymptotics on infinite-dimensional spaces. Note that this is not the case for finite-dimensional systems with regard to the significance of eigenvalues to the asymptotic behavior of such systems. However, as pointed out by a reviewer, misleading properties of spectra on the behavior of dynamical systems might occur in finite dimension with regard to their transient behavior. For instance, consider the two-dimensional discrete-time system

$$
x_{n+1}=A x_{n}
$$

with matrix $A$ given by

$$
A=\left(\begin{array}{cc}
0.90 & 1 / \epsilon \\
0 & 0.95
\end{array}\right)
$$

and $\epsilon>0$. The trajectories of this discrete-time system are given by the sequences $\left\{A^{n} x_{0}\right\}_{n \geq 0}$ for any initial state values $x_{0} \in \mathbb{R}^{2}$ and clearly $\lim _{n \rightarrow \infty}\left\|A^{n} x_{0}\right\|=0$. This system is asymptotically stable and hence s.c. However, the trajectories of this asymptotically stable system can lead to some period of growth very far away from the origin before they decay to zero as shown in Figure 6.1. This growth can be made arbitrarily large by letting $\epsilon \rightarrow 0$.

This finite-dimensional system has a transient behavior that differs entirely from the asymptotic behavior suggested by the spectrum (i.e., the eigenvalues). From an engineering point of view, such "almost unbounded" state trajectory behaves as that of a "practically unstable" system. Clearly, the spectrum of the state matrix (and hence a possible similarity to contraction of this matrix) fails as a tool for predicting the transient behavior of such finite-dimensional systems and therefore it is not a good indicator of this behavior. Further details which are beyond the scope of this paper can be found in $[8,13]$ and 


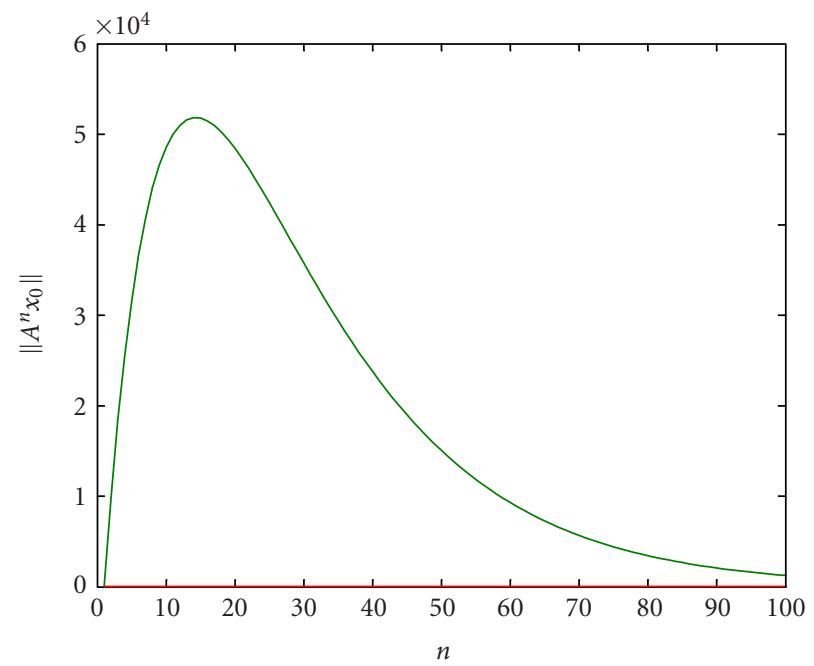

Figure 6.1. Transient growth of $A^{n} x_{0}$ with initial state $x_{0}=\left[\begin{array}{ll}1 & 1\end{array}\right]^{T}$.

the references therein, where particular mathematical tools, known under the name of pseudospectra, are used for the analysis of the transient behavior of linear systems.

\section{Appendix}

Let $r_{\sigma}(\mathscr{F})$ be the spectral radius of $\mathscr{F}$ as defined by $(2.5)$, then we have the following lemma on the "growth bound" of $\mathscr{F}^{n}$.

LEMMA 7.1. For $r>r_{\sigma}(\mathscr{F})$, there exists a constant $M_{r}>0$ such that

$$
\left\|\mathscr{F}^{n}\right\|_{\mathscr{B}(X)} \leq M_{r} r^{n}
$$

Proof. By the very definition of the spectral radius, if $r>r_{\sigma}(\mathscr{F})$, then there exists a positive integer $m$ such that $\left\|\mathscr{F}^{n}\right\|_{\mathscr{R}_{(X)}}^{1 / n}<r$ for $n>m$ or equivalently

$$
\left\|\mathscr{F}^{n}\right\|_{\mathscr{B}(X)}<r^{n} \text { for } n>m \text {. }
$$

But $\mathscr{F}^{n}$ is uniformly bounded on any finite interval, that is, for a fixed positive integer $m$, there exists a constant $M_{0}$ such that

$$
\left\|\mathscr{F}^{n}\right\|_{\Re(X)}<M_{0} \quad \text { for } 0 \leq n \leq m .
$$

Set $n=k m+l$, where $k$ and $l$ are positive integers and $0 \leq l \leq m-1$. Using the semigroup property of $\mathbb{F}$, we obtain

$$
\left\|\mathscr{F}^{n}\right\|_{\mathscr{B}_{(X)}} \leq\left\|\mathscr{F}^{k m}\right\|\left\|\mathscr{F}^{l}\right\| \leq M_{0}\left\|\mathscr{F}^{k m}\right\| \leq M_{0} r^{k m}=M_{0} r^{n-l}=\left(M_{0} r^{-l}\right) \cdot r^{n}
$$


Note that for $r \geq 1, M_{0} r^{-l} \leq M_{0} r^{-m} \leq M_{0}$ and we can take $M_{r}=M_{0}$ and for $r<1$, we have $M_{0}<M_{0} r^{-l} \leq M_{0} r^{-m}$ and we set $M_{r}=M_{0} r^{-m}$.

This lemma states that $\mathscr{F}^{n}$ does not grow faster than a "discrete exponential."

\section{Acknowledgment}

The author wishes to thank the reviewer who suggested the example motivating the remark in Section 6 about the misleading properties of eigenvalues on the behavior of dynamical systems even in the finite-dimensional case.

\section{References}

[1] R. A. Adams, Sobolev Spaces, Academic Press, New York, 1975.

[2] T. Chen and B. Francis, Optimal Sampled-Data Control Systems, Communications and Control Engineering Series, Springer-Verlag, London, 1996.

[3] S. R. Foguel, A counterexample to a problem of Sz.-Nagy, Proc. Amer. Math. Soc. 15 (1964), 788-790.

[4] B. A. Francis and T. T. Georgiou, Stability theory of linear time-invariant plants with periodic digital controllers, IEEE Trans. Automat. Control 33 (1988), no. 9, 820-832.

[5] P. R. Halmos, On Foguel's answer to Nagy's question, Proc. Amer. Math. Soc. 15 (1964), 791793.

[6] - A Hilbert Space Problem Book, D. Van Nostrand, Ontario, 1967.

[7] - Ten problems in Hilbert space, Bull. Amer. Math. Soc. 76 (1970), 887-933.

[8] D. Hinrichsen and A. J. Pritchard, On the Transient Behaviour of Stable Linear Systems, Proc. 14th International Symposium of Mathematical Theory of Networks and Systems (MTNS 2000), Perpignan, 2000, pp. 19-23.

[9] P. T. Kabamba, Control of linear systems using generalized sampled-data hold functions, IEEE Trans. Automat. Control 32 (1987), no. 9, 772-783.

[10] T. Kato, Perturbation Theory for Linear Operators, Springer-Verlag, Berlin, 1980.

[11] G. Pisier, A polynomially bounded operator on Hilbert space which is not similar to a contraction, J. Amer. Math. Soc. 10 (1997), no. 2, 351-369.

[12] D. L. Russell and G. Weiss, A general necessary condition for exact observability, SIAM J. Control Optim. 32 (1994), no. 1, 1-23.

[13] L. N. Trefethen, Pseudospectra of linear operators, SIAM Rev. 39 (1997), no. 3, 383-406.

[14] G. Weiss, Admissible observation operators for linear semigroups, Israel J. Math. 65 (1989), no. 1, $17-43$.

Joseph J. Yamé: Service d'Automatique et Analyse des Systèmes, Université Libre de Bruxelles, 1050 Bruxelles, Belgium

E-mail address: joseph.yame@ulb.ac.be 


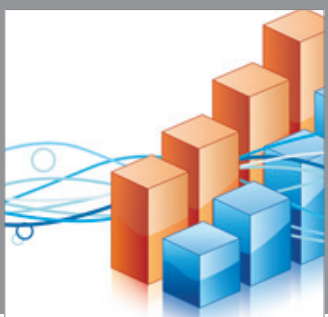

Advances in

Operations Research

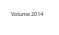

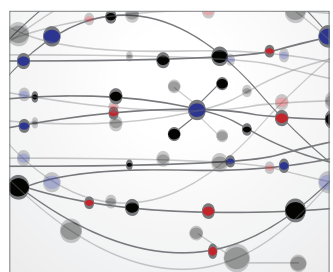

\section{The Scientific} World Journal
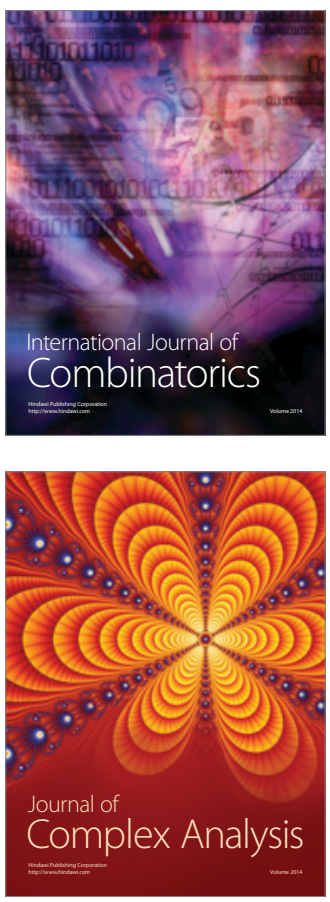

International Journal of

Mathematics and

Mathematical

Sciences
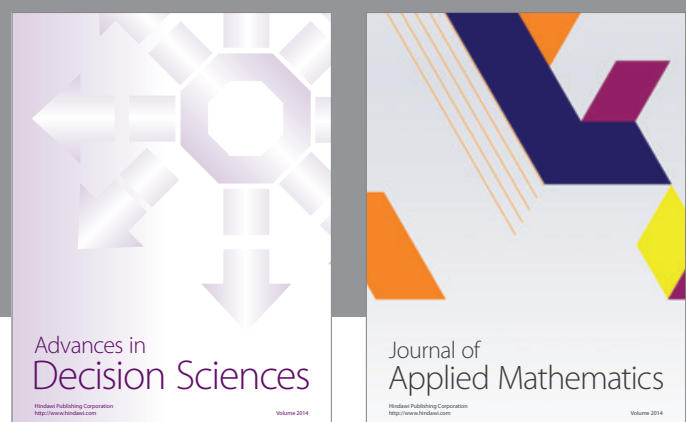

Journal of

Applied Mathematics
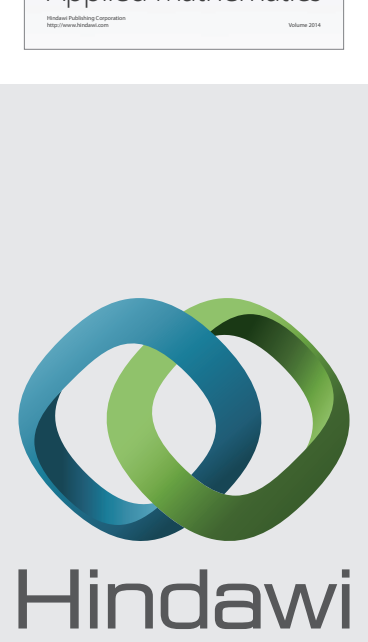

Submit your manuscripts at http://www.hindawi.com
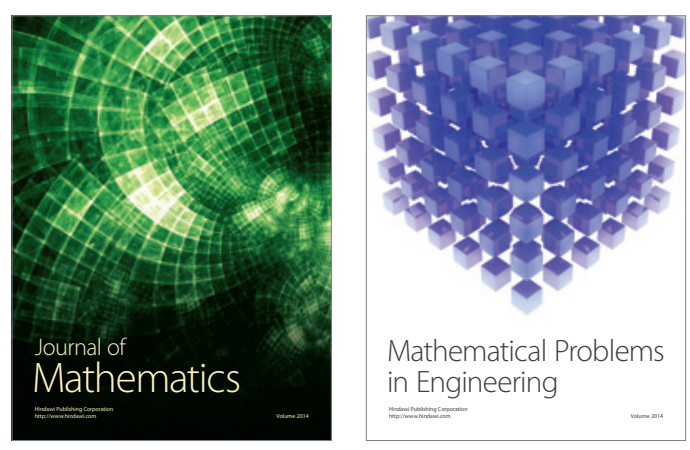

Mathematical Problems in Engineering
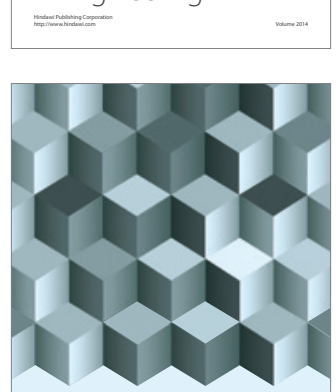

Journal of

Function Spaces
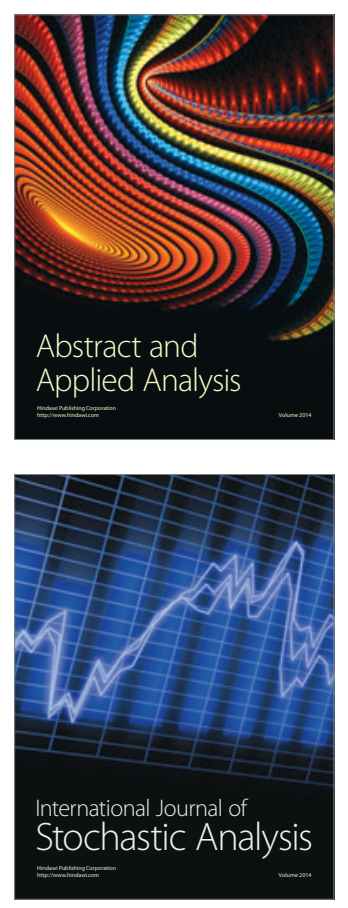

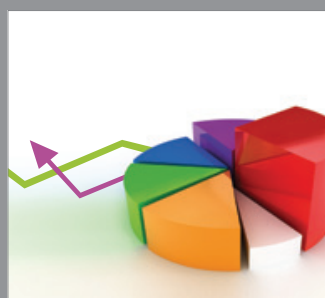

ournal of

Probability and Statistics

Promensencen
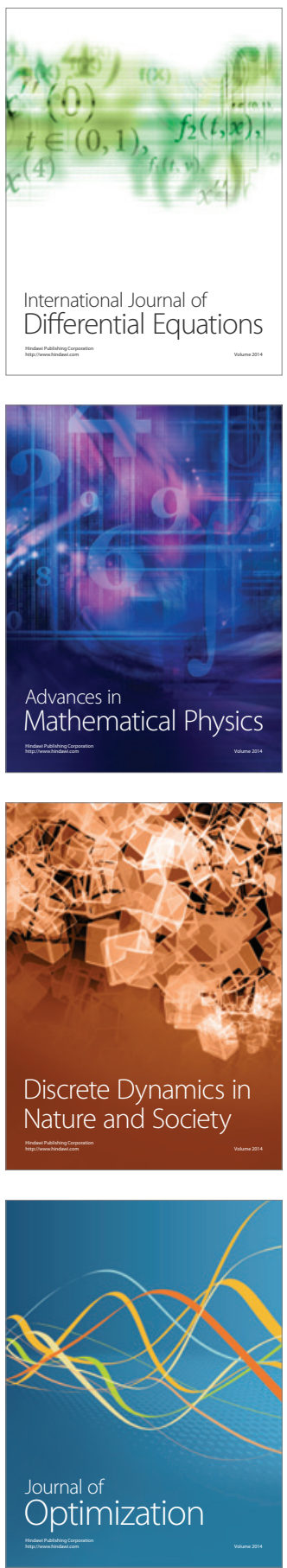